\title{
Bicycle Powered Mobile Phone Charger
}

\author{
Yip Winn Sheng Alwyn ${ }^{1}$ and Mohd Faizal ${ }^{1,2^{*}}$
}

${ }^{1}$ School of Computer Science and Engineering, Taylor's University Lakeside Campus, 47500 Subang Jaya, Selangor, Malaysia

${ }^{2}$ Solar Energy Research Institute, Universiti Kebangsaan Malaysia, 43600 Bangi, Selangor, Malaysia.

\begin{abstract}
The invention of electricity has become an essential part of every aspect of the society globally. With the increasing demand for the electrical appliance and electronic gadgets, this has also increased the consumption of energy significantly. As a result, we experience power outage which causes huge losses to industries. Hence, this research address if it is sustainable to use alternative energy in the form of bicycle power to charge a mobile phone. Bicycle power is a form of renewable energy that does not require the burning of fossil fuel to generate energy. The objective of this study is to design a mobile phone charger electrical circuit which consist of the integration of electrical components. Subsequently, the energy and power consumption between the conventional charging method and pedal powered charger can be assessed. This analysis is done to determine if pedal power is an economically sustainable solution to generate electricity. In this analysis, three selected dynamos hubs were assessed based on the performance data during low, medium, and high speed. The relationship between voltage, power speed, and time were obtained from the performance data and calculations. The chosen dynamo hub was the mid-range model, Shimano DH-3D72 which produces sufficient power at low and high speed to charge the mobile phone. The next analysis was the DC-DC converter topology which was essential to ensure that the output voltage was capped at $5 \mathrm{~V}$. The results have shown that a SEPIC converter was chosen based on the designed electrical circuit and application used. The third analysis was the selection of ultracapacitor which was needed to store energy during high speed and discharge energy during low speed. The selected ultracapacitor was justified with relevant calculations and further simulated in an analog circuit software, LTSpice XVII. All the selected electrical components were integrated to form a complete electrical circuit and were simulated under various inputs condition. The outcome of the results shown that the efficiency of the charging system was improved by $30 \%$. The payback period for this system would require only 406 days which is equivalent to 1.1 years.
\end{abstract}

\footnotetext{
* Corresponding author: drfaizalfauzan@gmail.com
} 


\section{Introduction}

In developing countries, technological advancement and modern transportation is rapidly shaping the world and our perspective for the future. This advancement plays a major role in improving productivity while reducing the cost of goods and services. At the same time, technology allows for faster and wider coverage of solutions to the economic, social, and environmental sector. However, the drawback of this advancement is the occurrence of pollution, the release of carbon emission excessively and causes people to be physically inactive and promotes many public health issues [1]. According to a statistical record, $94 \%$ of electricity generated in the country was by fossil fuels and this figure is expected to be unchanged over the next decade [2]. In addition, the energy consumption of the country has been increasing significantly over the last few decades because of an increased population. As a solution, many industries have been developing energy efficient technologies and ecofriendly products.

One of the sustainable ways to overcome this lingering issue is the usage of human powered devices. Human power has a few advantages that can be worthy of attention. One of the benefits of HPP from an eco-friendly design standpoint is they do not consume energy from non-renewable resources during their entire life span. Secondly, the benefit in having the ability power your device at any time is convenient. Thirdly, human also has the emotional benefit as this will enhance the quality of life by allowing them to feel more environmentally aware and more pro-active [3].

At present, bicycle powered mobile phone charger has not been commercialized due to the invention of power banks which is another portable form of charging mobile devices that is not considered in this research. However, there has been several products built which uses a generator or dynamo which converts rotation energy from pedaling a bicycle into electrical energy to charge the mobile phone. These products can charge the mobile phone but have certain limitations such as dynamo hub drag, low efficiency, insufficient power generated at low speed and high voltage produced at high speed. Hence, this study was done to analyze the integration of different components such as dynamo hub, DC-DC converter and ultracapacitor to generate sufficient power for charging at low speed and storing excess power generated at high speed. From there, the energy produced can be compared with a conventional charging method to determine if it is an economical sustainable solution to generate electricity.

\section{Key Design Elements}

The main concept of a bicycle powered mobile phone charger is to produce sufficient power to charge a mobile phone at any given speed with the integration of a dynamo hub, DC-DC converter and ultracapacitor. Ultracapacitor was chosen as it has a longer lifespan as compared to batteries. Additionally, it also has a long lifespan, high energy storage and fast charging and discharging time as compared to a regular capacitor which has been integrated in existing mobile phones charger. In this paper, the electrical circuit system is designed by using LTspice XVII, based on the performance data of the chosen component that affects the layout of the proposed circuit. The parameters are as following:

- Selection of Types of Dynamo Hubs and wheel combinations

- Expected output received by the mobile phone

- Type of DC-DC converter topology

- Efficiency of the system 
Subsequently, a simple economical study was conducted on the proposed design to determine the sustainability of this design. The electrical energy saved to break even the cost of production can be estimated.

\section{METHODOLOGY}

\section{1 selection of dynamo hub and wheel combination}

In this analysis, three types of dynamo hub and wheel combination are selected based on the different price range and wheel size which affects the dissipation of electrical energy. The first dynamo is the Panasonic Sanyo H27 on a 26" wheel which cost around \$70. This lowend dynamo hub provides up to $6 \mathrm{~V}$ at $3 \mathrm{~W}$ in power and is rated at $30 \%$ efficiency. The second dynamo is the popular Shimano DH-3D72 on a 28 " wheel which sits around $\$ 100$. This midrange dynamo hub is rated at $45 \%$ efficiency which enables it provides an output of $6 \mathrm{~V}$ at $3 \mathrm{~W}$ in power. The third dynamo is the Son Delux Dynamo on a 20" wheel which cost RM800. This high-end dynamo is rated at $65 \%$ efficiency which provides the same output power as the other two dynamos. Hence, the three dynamos require the same input power but have different efficiency. The voltage and output power of the dynamo hub is reliance on the speed of pedaling and load of the circuit.

The results for the three dynamo hubs which was tested under various speeds and loads were tabulated in a table and plotted into graphs as shown in the results below. In order to classify the dynamo hub as a power source, a few parameters such as voltage, power, electrical frequency, and subsequently electrical energy were calculated and tabulated. Equation (1) shows the calculation for electrical frequency of the dynamo hub, which is dependent on the speed of wheel and number of poles [4]. Subsequently, the power generated at different speeds can be calculated by using Equation (2) [4].

$$
\begin{aligned}
& F=\frac{p v}{120} \\
& P=V I
\end{aligned}
$$

Where, $\mathrm{p}=$ Number of poles, $\mathrm{v}=$ speed $(\mathrm{mph}), \mathrm{V}=$ voltage, and $\mathrm{I}=$ electric current.

The purpose of obtaining the electrical frequency and power is to determine at which speed the dynamo hub can charge the mobile phone at the required output parameters as shown in Table 1. When the electrical frequency is low, an input ultracapacitor will be needed to create a larger capacitance. Whereas, when the peak voltage is reached, a DC-DC converter must be used to lower the input voltage at the required parameters to prevent the damage of other electrical components.

Table 1. Output parameter of conventional charging of iPhone 6

\begin{tabular}{|c|c|}
\hline Output Parameter & Unit \\
\hline Voltage & 5V (Direct Current) \\
\hline Current & $0.5-1 \mathrm{~A}$ \\
\hline Power & $2.5-5 \mathrm{~W}$ \\
\hline
\end{tabular}




\subsection{DESIGN OF ELECTRICAL CIRCUIT}

The system is divided into four sub-system which is the dynamo hub itself, bridge rectifier, input ultracapacitor and DC/DC converter. First, alternating current (AC) is produced by the dynamo hub which is rectified by the bridge rectifier to Direct Current (DC). Subsequently, an input regulator is needed to reduce the voltage produced at high speeds and increase the voltage produced at low speed. In this study, when the speed increases, a high amount of voltage is produced which damaged other components such as the mobile phone itself. Hence, the converter is required to regulate the voltage to $5 \mathrm{~V}$ to ensure safe charging. Next, the bridge rectifier should consist four super barrier rectifiers (SBR) diodes. This model of diode was chosen as it is designed for high power and operates at low voltage at the forward bias terminal. For this design, a forward low voltage drop is preferred as the input voltage should perform at the peak to accommodate low cruising speed. The SBR diode also has a forward biased current of up of $1 \mathrm{~A}$ and a maximum current of 40A. Hence, it is suitable to withstand the repetitive current which consist of the capacitor peak current and the input current. Figure 1 displays a simple illustration of the design of electric circuit to produce DC current for the mobile phone.

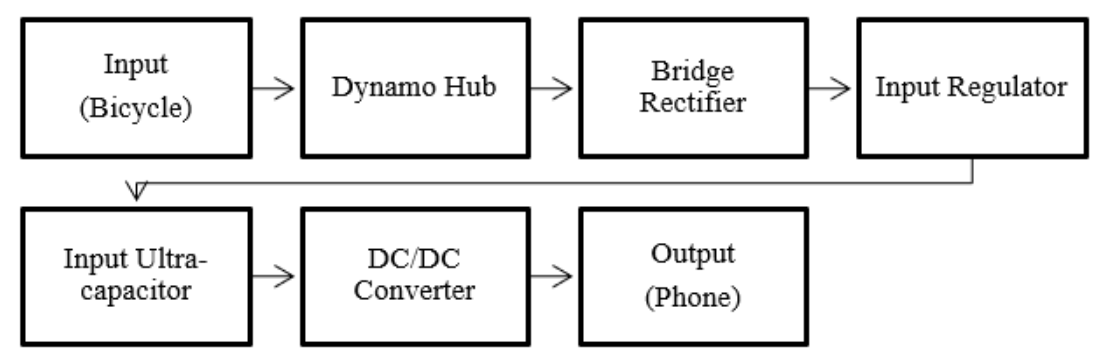

Fig. 1. Flow chart of electrical circuit.

\subsection{SELECTION OF DC-DC CONVERTER}

As mentioned above, the output DC voltage must be regulated to a minimum of $5 \mathrm{~V}$ to charge the iPhone 6 to prevent oversupply of voltage which will damage the mobile phone. DC-DC converter are commonly used to power electronics such as smart phones, media transmission and office equipment. Currently, most DC-DC converter such as the buck-boost converter, CUK converter, Single-Ended Primary Inductor Converter (SEPIC) and the fly back converter are designed to perform the requirement of specific applications [5]. These converters are built with only an active switch which is commonly MOSFET, a diode and an inductor [6]

Single-Ended Primary Inductor Convertor (SEPIC) was chosen as this converter takes in a range of input DC voltage and increases or decreases the output voltage. This can be done by controlling the duty cycle of a pulse to the MOSFET. In addition, the benefit of this convertor is that it does not invert the polarity of output voltage as well [7]. However, the usage of SEPIC will require extra parts which increases the power dissipation. Nonetheless, the output voltage required is only $5 \mathrm{~V}$ which makes this converter the best option for this application. The model of SEPIC converter considered in this analysis is the LT3759 due to the input range of $1.6 \mathrm{~V}$ to $49 \mathrm{~V}$ which is sufficient for this application. Before designing the circuit, a few parameters such as the input voltage, output voltage, and diode forward voltage must be determined. From these values, the duty cycle of the main switch can be obtained.

The maximum operational duty cycle of the converter is essential in increasing the output voltage. Conventionally, the maximum duty cycle, $\mathrm{D}_{\max }$ shall not be exceeded to prevent 
pulse skipping which leads to failure of the converter. Equation (3) shows the calculation to calculate the maximum duty cycle [5].

$$
D_{\text {max }}=\frac{V_{\text {out }}+V_{d}}{V_{\text {in (min) }}+V_{\text {out }}+V_{d}}
$$

Where, $\mathrm{V}_{\text {out }}=$ Output Voltage, $\mathrm{V}_{\mathrm{d}}=$ Diode forward voltage and $\mathrm{V}_{\mathrm{in}(\mathrm{min})}=$ Minimum Input Voltage.

In a SEPIC converter, it contains two inductors, L1 and L2 to store the energy from the input voltage. Equation (4) and equation (5) shows the calculation for the electric current that flows through the input conductor and output conductor, L1 and L2 [5].

$$
\begin{aligned}
& \mathrm{I}_{\mathrm{L} 1(\max )}=\mathrm{I}_{\text {in (max) }}=\mathrm{I}_{\text {out (max) }} \frac{\text { Dmax }}{1-\mathrm{D}_{\max }} \\
& \mathrm{I}_{\mathrm{L} 2 \text { (max) }}=\mathrm{I}_{\text {out (max) }}
\end{aligned}
$$

Where, $\mathrm{I}_{\mathrm{L} 1 \text { (max) }}$ is maximum current of inductor $1, \mathrm{I}_{\mathrm{L} 2 \text { (max) }}$ is maximum current of inductor $2, \mathrm{I}_{\text {out (max) }}$ is maximum output current and $\mathrm{D}_{\max }$ is maximum duty cycle.

The inductor value can be determined with equation (6) which is relating the operating frequency $\left(\mathrm{f}_{\mathrm{osc}}\right)$, input voltage $\left(\mathrm{V}_{\mathrm{in}(\mathrm{min})}\right)$ and the switch electric current $\left(\Delta \mathrm{I}_{\text {switch }}\right)$ [5]

$$
\mathrm{L} 1=\mathrm{L} 2=\frac{\mathrm{V}_{\text {in }(\min )}}{\Delta \mathrm{I}_{\mathrm{switch}} \cdot \mathrm{f}_{\mathrm{osc}}} \cdot \mathrm{D}_{\max }
$$

In the electrical circuit, a metal oxide semiconductor field effect transistor known as MOSFET is integrated into the electrical circuit. Equation (7) shows the calculation for the voltage rating of the MOSFET which has a safety margin by $10 \mathrm{~V}$ as recommended by the datasheet of LT3759 [5].

$$
\mathrm{V}_{\text {MOSFET }}=\mathrm{V}_{\text {Out(max })}+\mathrm{V}_{\text {in(max })}+10 \mathrm{~V}
$$

Where, $\mathrm{V}_{\text {MOSFET }}$ is voltage rating of MOSFET switch, $\mathrm{V}_{\text {Out(max) }}$ is maximum output voltage and $V_{\text {in(max) }}$ is maximum input voltage.

\subsection{Selection of Ultracapacitor}

In this application, the technology of ultracapacitor is integrated into the electrical system. The ultracapacitor functions to store the energy generated from the dynamo hub electrostatically. When the speed of wheel increases, high amount of voltage is generated which can be used to charge the ultracapacitor. The charged stored can act as a power reserve for low speed cycling when the voltage produced is insufficient to charge the mobile phone. Before selecting the specification of ultracapacitor, a few parameters such as the minimum and maximum input voltage of the circuit, output current, peak current, and total run time required for the mobile phone charging application must be identified. Every ultracapacitor is rated accordingly by the rated voltage, $V_{r}$ and is generally a low voltage device. As such, the rated voltage of the ultracapacitor is mostly lower than the intended voltage of the 
application. However, the maximum input voltage can determine the number of ultracapacitor cells which is required to be connected in series in the electrical circuit.

Referring to datasheet from Maxwell Technologies, the number of ultracapacitor connected in series can be calculated with Equation (8) [8].

$$
\text { Number of ultracapacitor, } N=\frac{V_{\text {in(max) }}}{V_{r}}
$$

Subsequently, an approximate capacitance of ultracapacitor required by the system can be determined with Equation (9) [8].

$$
\mathrm{C}_{\text {system }}=\mathrm{I} \cdot \frac{\mathrm{dt}}{\left(\mathrm{V}_{\mathrm{in}(\max )}-\mathrm{V}_{\mathrm{in}(\min )}\right)}
$$

The array of ultracapacitors can be arranged in series to ensure that it is able to accommodate the maximum input voltage. Equation (10) is to calculate the capacitance value for each ultracapacitor in series [8].

$$
\mathrm{C}_{\text {system }}=\frac{1}{\mathrm{C}} \cdot \mathrm{N}
$$

The obtained capacitance value is used to be compared with the data sheet of the chosen ultracapacitor model to ensure that it suits the application. However, if the ultracapacitor is insufficient to meet the input voltage, one or more of the ultracapacitor can be placed in parallel circuit. After selecting the suitable ultracapacitor, equation (11) shows the maximum energy stored, $\mathrm{E}_{\max }$ in the unit of watt per hour of the ultracapacitor chosen [8]. Where, $\mathrm{C}$ is capacitance of system, and $V_{r}$ is Rated voltage.

$$
E_{\max }=\frac{\frac{1}{2} C V_{r}^{2}}{3600}
$$

However, it is also worth mentioning that in the application of ultracapacitor, there are many other factors to consider in the sizing of capacitance. As such, the factors of internal resistance of the capacitor, ambient temperature and leakage current are not considered in this study.

\subsection{Efficiency of System}

The selected DC-DC converter and ultracapacitor model is then integrated into an electrical circuit and is simulated in LTspice XVII under a few variations of input voltage. The input voltage was obtained from the performance date of the chosen dynamo hub. As the input voltage produced is different from low, medium, and high speeds, many different simulations can be done. However, in this study, only the voltage produced at low speed and high speed is simulated. From the simulation, the electrical components of choice can be assessed if it has exceeded the components ratings calculated in the previous section.

Subsequently, the efficiency of the system which varies at different load for different input voltage supplied to the system. Ideally, the efficiency of the system should improve with the addition of the ultracapacitor and the DC-DC converter which enables the mobile phone to be charged efficiently at low speed and high speed. Equation (12) shows the calculation for the efficiency of the system [9]. 


$$
\text { Efficiency }=\frac{\text { Power Output }}{\text { Power Input }} \times 100 \%
$$

\subsection{Economic Viability}

In this study, the economic study was conducted to identify the initial cost needed to integrate a dynamo hub, DC-DC converter and ultracapacitor to generate sufficient power to charge a mobile phone. In addition, the cost of energy generated can also be calculated by using the local tariff rates to identify the savings from using this renewable source. Subsequently, the payback period for this system can be identified by using Equation (13) which equates the initial cost of the system and the cost of electricity saved on daily basis [10]

$$
\text { Payback Period }=\frac{\text { Initial Cost }}{\text { Cost of Electricity Saved per day }}
$$

\section{Results and Discussion}

\subsection{Performance data of dynamo hubs}

Since the minimum required input voltage was $5 \mathrm{~V}$, low speed performance was important in this analysis. Additionally, an average cyclist would be able to achieve a speed of between 0-10 mph. The dotted lines on the left in Figure 2 indicates the low speed range. At $7 \mathrm{mph}$, the Shimano DH-3D72, Son Delux and Sanyo H27 managed to produce 5.58V, 4.59V, and 3.3V respectively as shown in Figure 2. This would mean that only the Shimano DH-3D72 is able to charge the phone at this speed while the other two dynamo hubs would require to cycle at higher speed to begin charging the mobile phone. This is because the Shimano DH$3 \mathrm{D} 72$ has a respectable 26 poles and is more optimized for low speed and high-speed cycling [11].

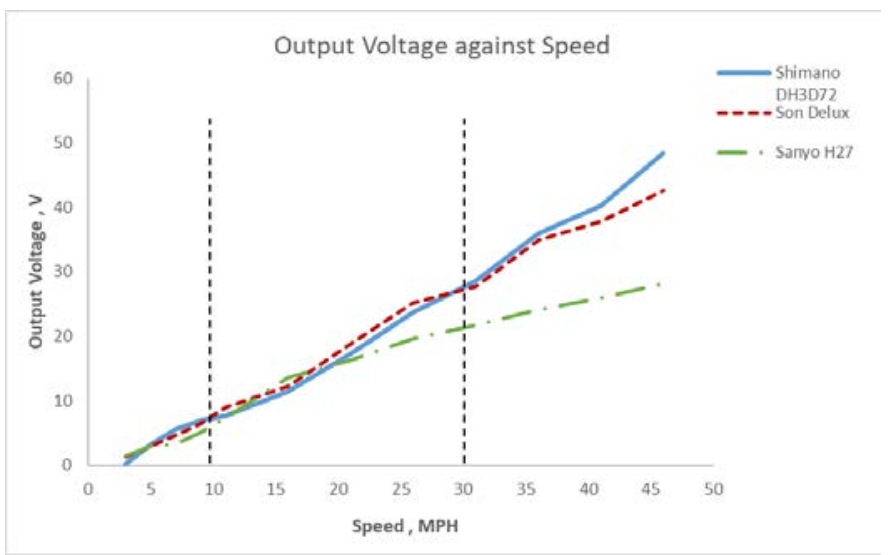

Fig. 2. Graph of output voltage against power

As for high-speed cycling, Shimano DH-3D72 managed to perform better than the highend Son Delux and Sanyo H27. At 40-45 mph, the Shimano DH-3D72 produces 23-28V as compared to Son Delux which produces $25-27 \mathrm{~V}$. The peak voltage produced by the medium 
end and high-end dynamo are $48.4 \mathrm{~V}$ and $42.56 \mathrm{~V}$, respectively. This peak voltage was used as input voltage for the design of electrical circuit in the selection of DC-DC converter below.

\subsection{Design of Electrical Circuit}

Since the peak voltage is high, a SEPIC converter was used to decrease the output voltage to ensure a constant $5 \mathrm{~V}$ output voltage. This converter is chosen as it does not invert the polarity of the output voltage. The electrical circuit was constructed in LTspice XVII based on the application of the SEPIC converter and the calculated electrical components ratings as shown in Table 2 .

Table 2. List of components in SEPIC topology electric circuit

\begin{tabular}{|c|c|c|}
\hline Components & Value & Rating \\
\hline MOSFET & - & $63.43 \mathrm{~V}, 5.1 \mathrm{~A}$ \\
\hline Sense Resistor & $9.8 \mathrm{~m} \Omega$ & - \\
\hline Rectifier Diode & - & $48.34 \mathrm{~V}, 1 \mathrm{~A}$ \\
\hline DC Coupling Capacitor & $4.5 \mu \mathrm{F}$ & $50 \mathrm{~V}$ \\
\hline Output Ultracapacitor & $10.13 \mathrm{~F}$ & $5 \mathrm{~V}$ \\
\hline Inductors & $4.05 \mu \mathrm{H}$ & $3.45 \mathrm{~A}$ \\
\hline
\end{tabular}

When primary switch is powered, energy produced from the dynamo hub is stored in the input inductor, L1 and output inductor, L2 which is connected by a coupling capacitor as shown in Figure 3. When the MOSFET is opened, energy is transferred through the coupling capacitor, directing the rectifier diode and to the output capacitor. The output voltage is then used to charge the mobile phone.

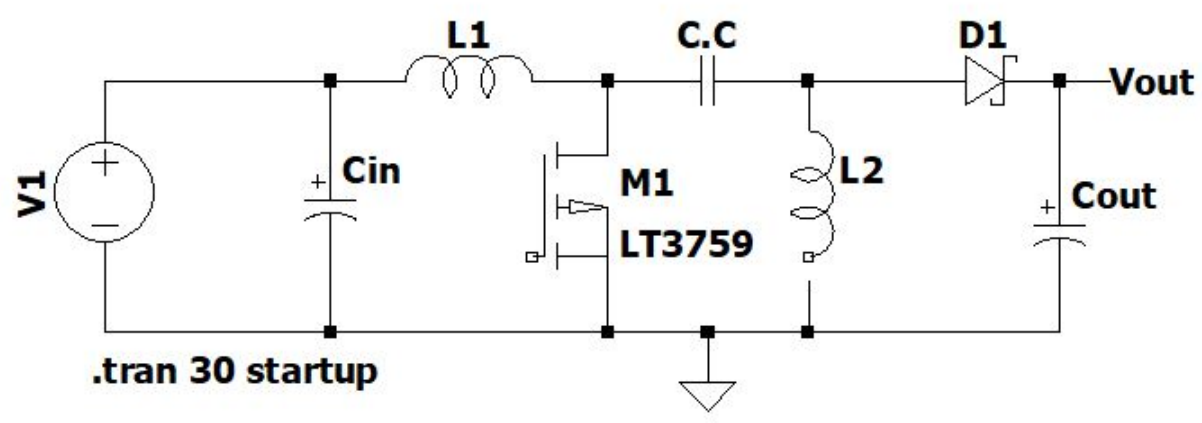

Fig. 3. Simple SEPIC topology illustration.

However, an issue arises when designing two separate inductors in a SEPIC converter electrical circuit whereby the two inductors with separate capacitors form a resonant circuit. Resonant circuit happens when the capacitance and inductances combine at a specific electrical frequency to produce maximum amplitude [12]. This would mean that the voltage increases to twice the applied voltage on a base transient. This increases the input voltage at high speed which can damage the components in the circuit or the mobile phone itself. As such, resistors are needed to be in series with the inductors. In addition, the inductors are also of the same inductance value to prevent a surge of voltage. The inductors are now coupled on the core and the energy is coupled through the SEPIC converter as seen in Figure 4. 


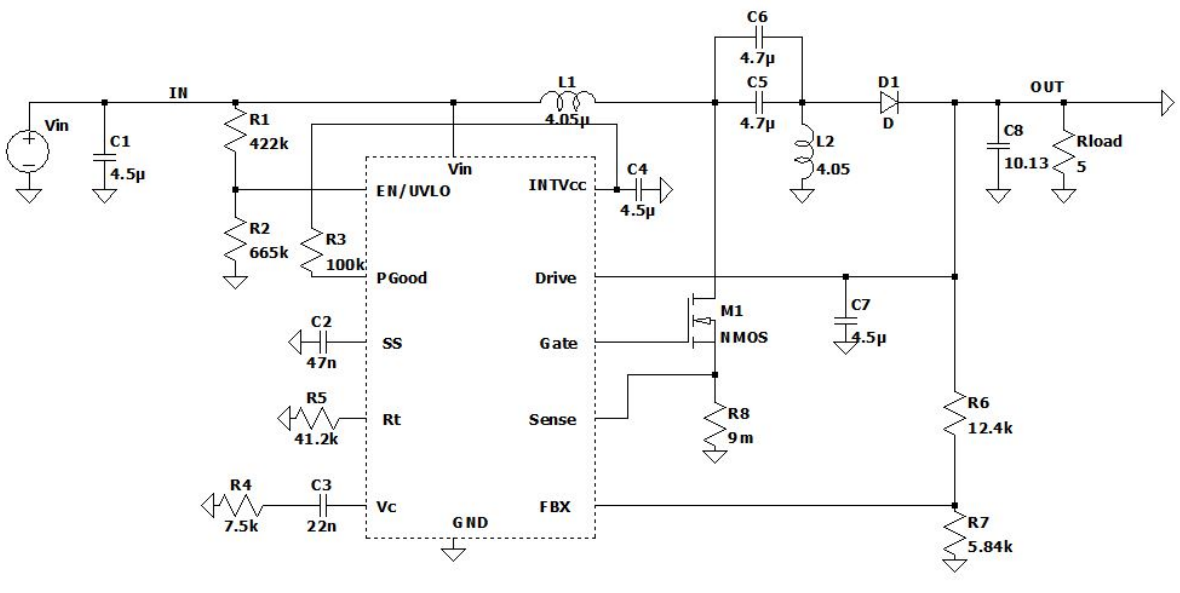

tran $30 \mathrm{~m}$ startup

Fig. 4. Simulation electrical circuit in LTSpice XVII

The most significant simulation results of the chosen dynamo hub with the SEPIC and chosen ultracapacitor would be at high speed. The input voltage was set between $22 \mathrm{~V}$ to $23 \mathrm{~V}$ which is produced at $23-25 \mathrm{mph}$. Whereas, the ultracapacitor was placed in series with an overall system capacitance of $10.13 \mathrm{~F}$. The rated voltage was calculated at $5 \mathrm{~V}$. The maximum energy stored in the unit of watt per hour was found to be $0.141 \mathrm{Wh}$. The simulation results as shown in Figure 5 were found to produce a consistent output voltage of $5 \mathrm{~V}$ with a maximum electric current of $1 \mathrm{~A}$ which further justifies the chosen electrical components ratings in this electrical circuit. As for the exact values of the resistors, it is simulated by the software itself and was not considered in this study.

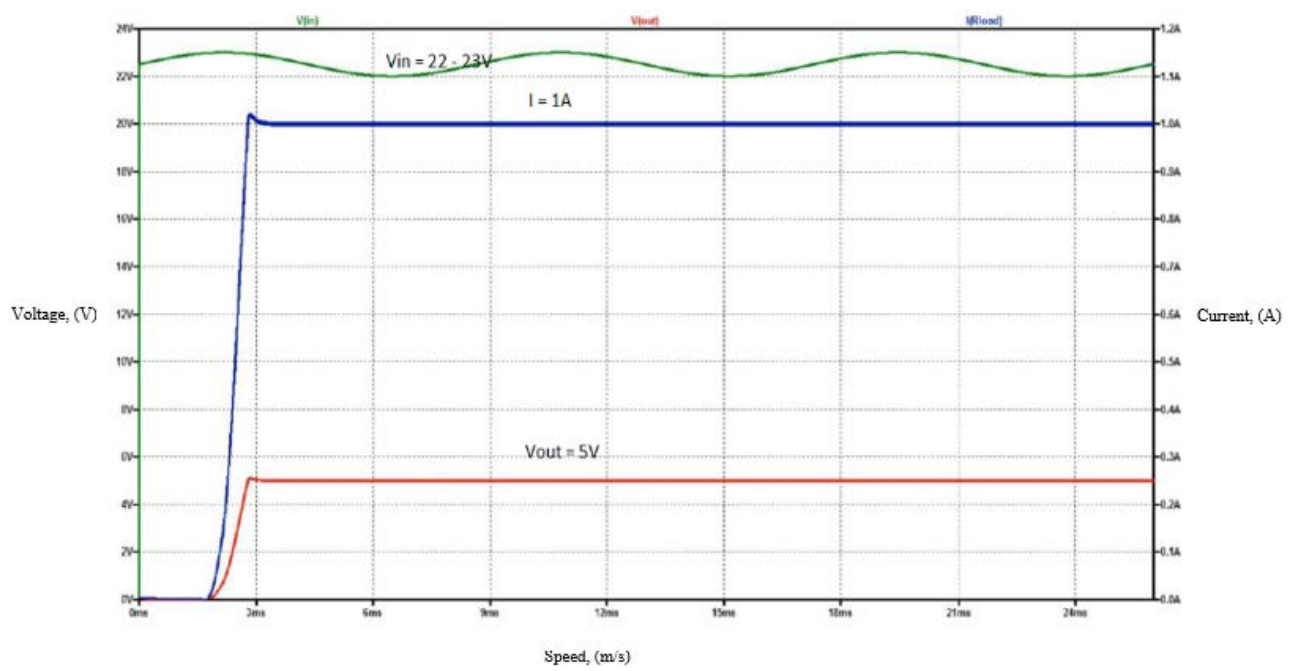

Fig. 5. Simulation results at high speed

As mentioned before, the rated efficiency of the chosen dynamo hub, Shimano DH-3D72 was $45 \%$. However, after integrating the SEPIC converter and ultracapacitor, the efficiency at $5 \mathrm{~V}$ output voltage was found to be higher at most of the speeds tested as shown in Figure 6 . In this test, the input voltage at $8 \mathrm{mph}, 10 \mathrm{mph}, 15 \mathrm{mph}$, and $25 \mathrm{mph}$ were tested with an increment of $0.1 \mathrm{~A}$ from no load condition to full load condition which was at $1 \mathrm{~A}$. The highest 
efficiency was recorded at $5.5 \mathrm{~V}$ input voltage and $0.5 \mathrm{~A}$ load with $82 \%$. This shows that at low speed the energy stored in the ultracapacitor is sufficient to compensate for the low input voltage to produce an output voltage of $5 \mathrm{~V}$ to charge the mobile phone. As for high speed, the efficiency is slightly lower at around $76-77 \%$. Overall, it is proven that the efficiency can be improved with the proposed system by about $30 \%$ as compared to a direct conversion of energy by using a dynamo hub itself.

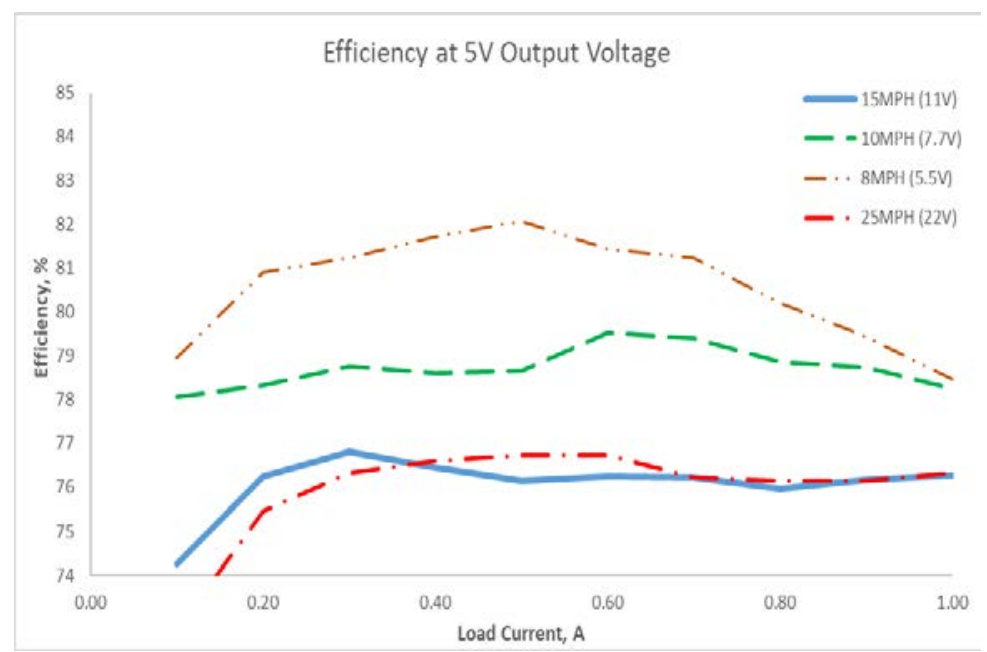

Fig. 6. Efficiency of system at $5 \mathrm{~V}$ output voltage.

\subsection{Economical Study}

The initial cost of this system includes the dynamo hub, components in the SEPIC topology and the chosen ultracapacitor model which is determined as shown in Table 3.

Table 3. Initial Cost of System

\begin{tabular}{|c|c|}
\hline Components & Cost \\
\hline Shimano DH-3D72 & RM369.75 \\
\hline DC Coupling Capacitor $4.5 \mu \mathrm{F}$ & RM12.00 \\
\hline Ultracapacitor $10.13 \mathrm{~F}, 10 \mathrm{~V}$ & RM50.00 \\
\hline MOSFET Gate Diode $0.15 \mathrm{~A}, 100 \mathrm{~V}$ & RM4.00 \\
\hline Sense Resistor $9.8 \mathrm{~m} \Omega$ & RM2.00 \\
\hline Inductor $4.05 \mu \mathrm{H}$ & RM4.00 \\
\hline Rectifier Diode $1 \mathrm{~A}$ & RM0.50 \\
\hline Total Initial Cost & RM442.25 \\
\hline
\end{tabular}

The selected dynamo hub consists of $83.6 \%$ of the initial cost of the whole system. With that, it is assumed that an individual cycles at an average speed of $20 \mathrm{mph}$ for 1 hour daily. The power generated would be equivalent to $5 \mathrm{kWh}$. The local tariff for the first $200 \mathrm{kWh}$ is rated at 21.80 cents which is multiplied with the power generated from cycling. 
As such, the cost of electricity saved is found to be RM1.09/day and an expected annual savings from the bicycle powered mobile phone charger would be RM397.85. The return of investment for this system is estimated at 406 days which is equivalent to 1.1 years. Thus, this proves that the proposed system integration into an existing dynamo hub is more energy efficient and less costly while still generating sufficient power to charge a mobile phone without relying on any power grid.

\section{Conclusion}

Overall, this paper acknowledged the capabilities of generating electricity from a bicycle by the integration of dynamo hub, converter and ultracapacitor technology. An analysis of the power generated by the dynamo hub and in-depth electrical circuit design has been integrated to produce a higher efficiency mobile phone charging system. The factors which was considered in this analysis was the selection of dynamo hub, DC-DC converter and ultracapacitor. The selection of dynamo hub was based on the performance data generated during low, medium, and high speed. As for the converter, the polarity and buck-boost function were considered to match the application of mobile phone charging. Whereas the ultracapacitor was selected based on relevant calculations to determine the rated capacitance and voltage. After obtaining the ratings of the electrical components, the electrical circuit was simulated on LTSpice XVII which is an analog circuit software to identify the output parameters of the circuit. From the simulation, it was found that the efficiency of the charging system improved by $30 \%$ as compared to a direct conversion of energy by using a dynamo hub itself which is considerably good.

Subsequently, a simple economic study was constructed to determine the cost of electricity saved based on local tariff rates. As a result, it was found that $5 \mathrm{kWh}$ can be generated on an average speed of $20 \mathrm{mph}$ for 1 hour daily. This would be a savings of RM1.09 per day and an annual savings of RM397.85. The payback period for this system would require only 406 days which is equivalent to 1.1 years.

All in all, this analysis has met the objectives of this research which was to justify that bicycle powered is an economical sustainable solution in the application of mobile phone charging. In addition, the analysis also provides more opportunities for future integrations of electrical components to improve the efficiency of the system and cost of production.

\section{References}

1. A. J. Jansen, A.L.N. Stevels, in J. Clean. Prod. 14, 1299 (2006).

2. K.M. Poh, H.W. Kong, J. Renew. Energy. 6, 3 (2002).

3. T. Dean, The Human-Powered Home: Choosing Muscles Over Motors. (New Society Publishers, British Columbia, 2008).

4. B. Rubini, K. Sasikala, D. Ravikumar, J. Eng. Appl. Sci. 12, 55 (2017).

5. M.S. Ramkumar, A. Amudha, P. Nagaveni, G. Emayavaramban, S. Divyapriya, V. Mansoor, Mater. Today. 3, 45 (2020)

6. S. Farhani, E. Barhoumi, F. Bacha, Ain. Shams. Eng. J. 2, 67 (2020).

7. S. Mouslim, M. Oubella, M. Kourchi, M. Ajaamoum, Mater. Today. 27, 3199 (2020).

8. Y.F. Yuan, L.L. Guang, Y.Y. Ping, H.Y. Sheng, GMRDC, 4, 3 (2010).

9. B. Keith, MHD. 55, 193 (2019).

10. H. Louie, K. Peng, E. Hoffstetter, S.J. Szablya, NAPS. 2, 18 (2010).

11. V. Kumar, P. Verma, MSEJ, 2, 45 (2015).

12. M. Orsic, D. Krajcinovic, IEEE Trans. Circuit Theo. 19, 533 (1972). 